\title{
CHARACTERIZATION OF THE FORMATION OF FILTER PAPER USING THE BARTLETT SPECTRUM OF THE FIBER STRUCTURE
}

\author{
MARTIN LEHMANN ${ }^{1}$, JOBST EISENGRÄBER-PABST ${ }^{1}$, JOACHIM OHSER ${ }^{\bowtie, 2}$ AND ALI \\ MOGHISEH $^{2}$ \\ ${ }^{1}$ Mann+Hummel GmbH, Hindenburgstr. 45, D-71638 Ludwigsburg, Germany; ${ }^{2}$ Univ. Appl. Sci. Darmstadt, \\ Dept. Math \& Nat. Sci., Schöfferstr. 3, D-64295 Darmstadt, Germany \\ e-mail: martin.lehmann@mann-hummel.com, jobst.eisengraeber-pabst@mann-hummel.com, jo@h-da.de, \\ ali.moghiseh@h-da.de
}

(Received December 20, 2012; revised May 22, 2013; accepted June 10, 2013)

\begin{abstract}
The formation index of filter paper is one of the most important characteristics used in industrial quality control. Its estimation is often based on subjective comparison chart rating or, more objective, on the power spectrum of the paper structure observed on a transmission light table. It is shown that paper formation can be modeled by means of Gaussian random fields with a well-defined class of correlation functions, and a formation index which is derived from the density of the Bartlett spectrum estimated from image data: the mean of the Bessel transform of the correlation function taken for wave lengths between 2 and $5 \mathrm{~mm}$. Furthermore, it is shown that a considerable variation of the local grammage can be observed also in cases where the the fibers are uniformly and independently scattered in the paper sheet.
\end{abstract}

Keywords: Bartlett spectrum, chart cloudiness, fiber system, filter paper, formation, image analysis.

\section{INTRODUCTION}

Filter papers are used in a wide variety of fields, ranging from air to oil filters (Durst et al., 2007). They consist of fibers, which are more or less randomly distributed. Except the specific paper weight (i.e., the weight per unit area, also called the nominal grammage), the weight distribution is a very important characteristic of paper. It influences many properties of filter papers such as flow rate, particle collection, efficiency, wet strength, porosity and dust holding capability. Thus, characterization of the weight distribution is important for industrial quality control as well as for the development of new filter materials and technologies of manufacture.

It is easy to get an impression of the weight distribution when holding a sheet of paper up against light and observing the distribution of the optical density, known as the paper formation, chart cloudiness or flocculation. Assuming a constant absorption coefficient for the solid constituents of the paper structure, the local intensity of the transmitted light can be related to the local weight density by Lambert-Beer's law. As a consequence, there is a close relationship between weight distribution and formation and, in fact, often one does not distinguish between both. See Van den Akker (1949), McDonald et al. (1986) and Lien and Liu (2006) for the computation of the grammage from the absorption of visible light. The use of soft X-radiation is suggested in
Farrington (1988), and the influence of the choice of radiation on transmittance is investigated for nonwoven fabrics in Boeckerman (1992) and for paper in Norman and Wahren (1976) and Bergeron et al. (1988).

Usually, the formation is experimentally determined based on two-dimensional (2D) images of the paper structure. A transmission light table is used in order to ensure a homogeneous illumination and the images are acquired by a CCD-camera having a linear transfer function, such that the pixel values can be assumed to be approximately proportional to the corresponding local intensities. In the simplest case, a paper structure inspection can be based on subjective comparison chart rating, supported by an industrial standard consisting on well-formulated rules for image acquisition and rating. Nevertheless, the valid industrial norm on paper, board, pulps and related terms gives only a rough description of the terms 'formation' (manner in which the fibers are distributed, disposed and intermixed to constitute the paper) and 'lock-through' (structural appearance of a sheet of paper observed in diffuse transmitted light), ISO 4046(E/F), 2012. Inspection systems based on image analysis include computation of a (more or less objective) value for a 'formation index' (or a 'formation number') from the image data. One should keep in mind that such a formation index estimated from image data is (widely) independent of the nominal grammage as well as on the variance of the 
local paper weight. But what is exactly meant by the 'formation index', and is it sufficient to characterize paper formation by only one number?

There is a huge number of publications on the characterization of paper formation (Kallmes, 1984; Cresson, 1988; Cherkassky, 1999; Drouin et al., 2001), see also Waterhouse et al. (1991) and Praast and Göttsching (1991) for a very good survey on literature from the late 1980th and Chinga-Carrasco (2009) for newer developments. An intuitive characteristic for the formation is the mean paper flock size, going back to Robertson (1956), but until now there is no convincing method for segmenting flocks in gray-tone images. More useful methods are based on measuring the variance of the pixel values or, more general, the co-occurrence matrix of the image data (Yuhara et al., 1986; Cresson, 1988; Cresson and Luner, 1990a;b). The approach presented in Pourdeyhimi and Kohel (2002) is motivated by a Poisson statistics for the centers of paper flocks (objects). On the one hand, these centers cannot safely be detected and, on the other hand, the computation of the 'uniformity intex' of the paper formation is based on the variation of the area fraction in a binarized image but even not on the flock centers.

In the carefully written monograph of Deng and Dodson (1994) a formation number $n=$ $\operatorname{var}_{r} / \operatorname{var}_{0}$ is defined as ratio of variances $\operatorname{var}_{r}$ and $\operatorname{var}_{0}$ of integrated local grammage, where the integration is over a square of edge length $1 \mathrm{~mm}$. In this approach, $\operatorname{var}_{r}$ is the variance for the real structure and $\operatorname{var}_{0}$ is the variance for a reference model (a Boolean segment process). This approach goes back to earlier works, e.g. Norman and Wahren (1974) and is frequently cited also in current literature, e.g., Sampson (2009). A similar approach is used in Farnood et al. (1995), where the fluctuation of the local grammage is modeled as a Poisson shot noise process (or a dilution model) of sperical flocks. I'Anson and Sampson (2003) discussed the relationship between Farnood's flocculation characteristic and the spectral density of paper formation.

Since woven textiles have a (more or less) periodic pattern, it seems to be obvious to apply Fourier methods for quality inspection, see e.g., Wang et al. (2011) and references therein, where slight deviations from the periodicity are detected based on the correlation function of the pattern or, analogously, its counterpart in the inverse space - the so-called power spectrum. The use of Fourier transform for an identification of periodic patterns in paper and board traces back to I'Anson (1995). In Sara (1978), Norman (1986), Cresson (1988), Provatas et al. (1996), Cherkassky (1998) and
Lien and Liu (2006), the correlation function and the power spectrum are also suggested as characteristics for cloudiness of (non-periodic but macroscopically homogeneous) nonwovens and paper formation, respectively (Section 2.2 in Alava and Niskanen, 2006). Sometimes the range of interaction, i.e., integral of the correlation function (also known as the integral range), is used as a formation index. Instead of a Fourier transform, Scharcanski (2006) uses a wavelet transform to extract a spectral density from the sheet formation.

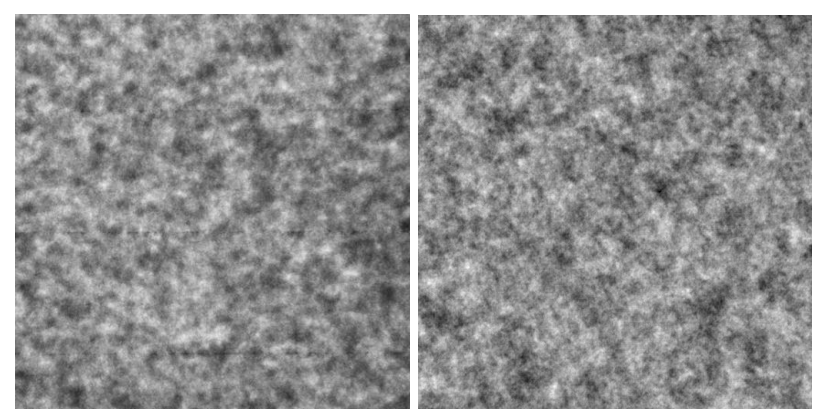

Fig. 1. An image showing the formation of a filter paper (left) and a realization of a macroscopically homogeneous and isotropic GRF (right) with $k(x)=$ $\mathrm{e}^{-\lambda\|x\|}$ and $\lambda=0.6 \mathrm{~mm}^{-1}$; the edge length of the images $102.4 \mathrm{~mm}$.

Mathematical modeling of paper structure on a mesoscale can lead to a deeper understanding, e.g., of the phenomenon of formation (Cresson, 1988; Cherkassky, 1998; Antoine, 2000; Gregersen and Niskanen, 2000; Provatas et al., 2000; Sampson, 2009), where the model parameters - so far they can easily be estimated from image data serve as formation characteristics. Further approaches are based on modeling random structures by Markow Random Fields (MRF) and decomposing the image of the structure into "different scales", evaluating the degree of homogeneity on each scale and computing an overall degree of homogeneity. Scholz and Claus (1999) applied this approach originally on the structure of nonwovens (fleeces and felts), but in principle this works also for the evaluation of paper structures, where the degree of homogeneity can be seen as a formation index. Notice that the "different scales" mentioned above are also known as the Laplacian pyramid of image data (Burt and Adelson, 1983).

In the present article we use Gaussian Random Fields (GRFs) for modeling paper formation and, following the suggestion made in $\mathrm{Xu}$ (1996) and Lien and Liu (2006), a Fourier approach is applied for computing characteristics of paper formation. More precisely, we show that the formation of the investigated filter papers can be characterized by the density of the Bartlett spectrum, i.e., a spectral representation of the correlation function, 
which can be estimated by the method of Koch et al. (2003). Using a parametric approach for the Bartlett spectrum, we introduce one of its parameters as a characteristic for paper formation. Nevertheless, to be independent of the fitting of a theoretical function to the experimental data, the formation index is determined directly from estimates of the spectral density.

Finally, it is shown by an example that the spectral density of the paper structure can contain high fractions of long waves even if no flocculation occurs, i.e., the fibers are uniformly and independently scattered in the paper sheet.

\section{MODELING PAPER FORMATION BY GAUSSIAN RANDOM FIELDS}

Visual inspection of Fig. 1 shows that the formation of filter paper is surely one of the most convincing applications for GRFs. The difference among the real structure on the left-hand side and the realization on the right-hand side, which is obtained from the adapted GRF, can be recognized only by experts. This is very important since, if formation can really be modelled by GRFs, then the Bartlett spectrum of a GRF uniquely specifies formation.
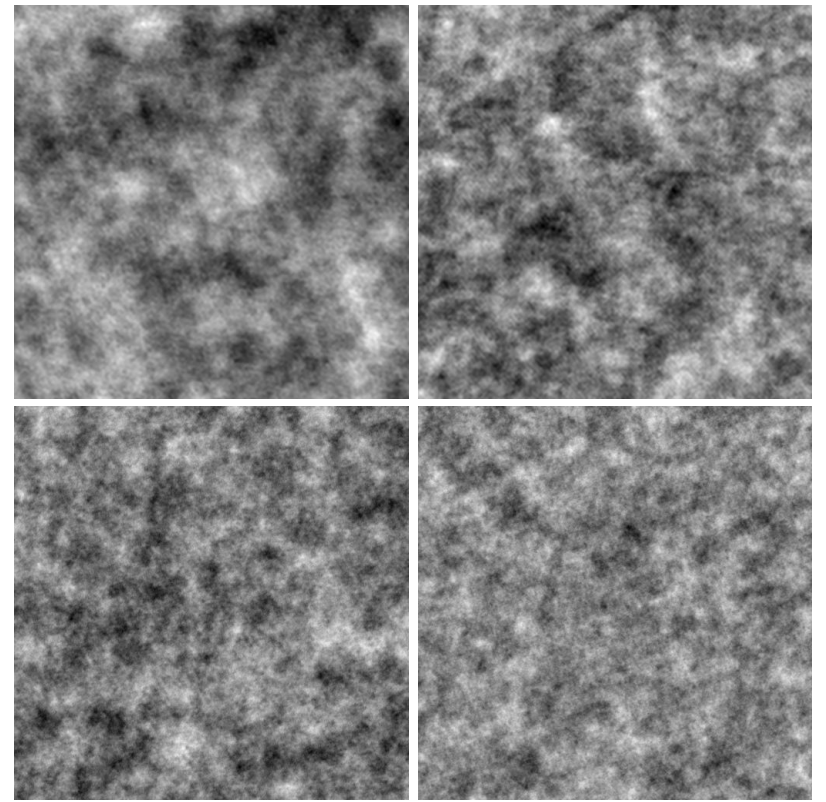

Fig. 2. Realizations of macroscopically homogeneous and isotropic GFRs for constant $\mu$ and $\sigma^{2}$ and exponential correlation function $k(x)=\mathrm{e}^{-\lambda\|x\|}$ with parameters $\lambda=0.1 \mathrm{~mm}^{-1}, \ldots, \lambda=0.4 \mathrm{~mm}^{-1}$, lexicographic order. The edge length of the images is $102.4 \mathrm{~mm}$.
As it is well known, a random field $\Phi(x)$ is a 2-dimensional, real-valued random function. The function $\Phi(x)$ is called macroscopically homogeneous (or stationary in the strict sense), if $\Phi(x)$ is invariant with respect to translations, i.e., its finite-dimensional distributions are translation invariant. Furthermore, $\Phi(x)$ is said to be isotropic, if it is invariant with respect to rotations around the origin. Finally, a random field $\Phi(x)$ is a Gaussian Random Field (GRF) if all its finite-dimensional distributions are multivariate normal distributions (see, e.g., Adler, 1981; Abrahamsen, 1985 and Adler and Taylor, 2007 for introductions to GRFs).

A macroscopically homogeneous GRF $\Phi(x)$ is uniquely specified by its mean $\mu=\mathbb{E} \Phi(x)$, its variance $\sigma^{2}=\mathbb{E} \Phi^{2}(x)-\mu^{2}$, and the covariance function

$$
\operatorname{cov}(x)=\mathbb{E}((\Phi(y)-\mu)(\Phi(y+x)-\mu)), \quad x \in \mathbb{R}^{2},
$$

which is independent of $y \in \mathbb{R}^{2}$ and positiv definite. The normalized function $k(x)=\operatorname{cov}(x) / \sigma^{2}$ is known as the (auto-) correlation function. The expectation $\mu$ is the first order characteristic of $\Phi(x)$ while $\sigma^{2}$ and $k$ are second order characteristics. All higher order characteristics depend only on $\mu, \sigma$ and $k$. This is a direct consequence of the Gaussianity (Abrahamsen, 1985), which means that our attention can be payed exclusively on the first and second order characteristics and their estimation.

Four realizations of GRFs $\Phi_{\lambda}(x)$ with an exponential correlation function $k(x)=\mathrm{e}^{-\lambda\|x\|}, x \in$ $\mathbb{R}^{2}$, are shown in Fig. 2. It turns out that the distributional properties of $\Phi_{\lambda}(x)$ distinguish by the positive scaling parameter, i.e., it holds $\Phi_{\lambda}(x) \stackrel{d}{=}$ $\Phi_{1}(\lambda x)$, and realizations of $\Phi_{\lambda}(x)$ can be obtained from realizations of $\Phi_{1}(x)$ by scaling.

If a GRF is well adapted to the image data of a paper structure, then the interpretation of its characteristics $\mu, \sigma^{2}$ and $k$ is as follows: The mean $\mu$ is the brightness, $\sigma$ corresponds to the image dynamics, and $k$ is the correlation function of the pixel values. Under some technical conditions (using of a CCD camera allowing photometric measurements, high gray-tone resolution, constant gain, etc.), assuming Lambert-Beer's law for light absorption and knowing the initial light intensity, the nominal paper grammage and the weight variance can roughly be estimated from $\mu$ and $\sigma^{2}$, respectively. As a consequence, the correlation function $k$ characterizes the paper formation uniquely. Fig. 3 shows realizations of two GRFs with the same $\mu$ and $k$ but different $\sigma$. One feels subjectively that the formation is the same in both images. 

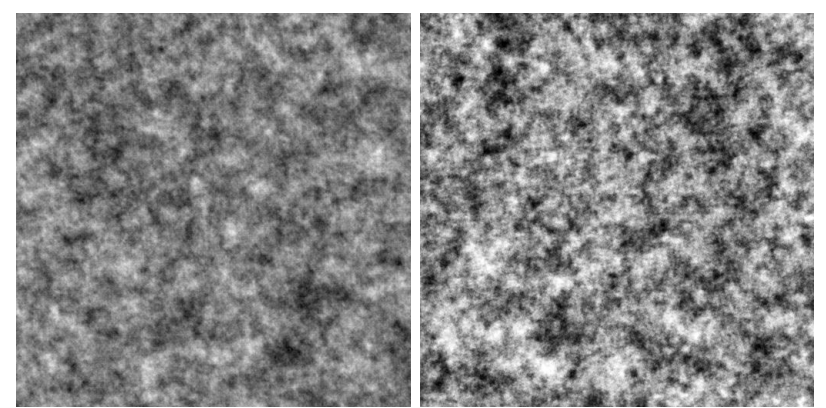

Fig. 3. Realizations of two macroscopically homogeneous and isotropic GRFs with constant $\mu$ and $k(x)=\mathrm{e}^{-\lambda\|x\|}$ with $\lambda=0.5 \mathrm{~mm}^{-1}$; left: small $\sigma$, right: larg $\sigma$. The edge length of the images is $102.4 \mathrm{~mm}$.

Finally, we remark that in the isotropic case the correlation function $k$ depends on only the radial coordinate $r=\|x\|$ of $x$, i.e., there is a function $k_{1}$ such that $k(x)=k_{1}(\|x\|)=k_{1}(r)$. Clearly, the exponential correlation function mentioned above is such a function.

\section{THE SPECTRAL REPRESENTATION OF THE CORRELATION FUNCTION}

First of all, we recall Bochner's theorem which states that the covariance function $\operatorname{cov}=\sigma^{2} k$ of a macroscopically homogeneous random field $\Phi(x)$ can be represented by a non-negative, bounded measure $\Gamma_{\Phi}$ - the so-called spectral measure or the Bartlett spectrum of $\Phi(x)$. A proof is given e.g., in Katznelson (2004), p. 170. This important theoretical result is also useful in applications, since efficient Monte Carlo techniques of generating realizations of GRFs as well as fast algorithms for estimating their second order characteristics are based on spectral representations. In this article we restrict ourselves to the particular cases in which the Bartlett spectrum $\Gamma_{\Phi}$ has a density $\gamma_{\Phi}$, which is also known as the spectral density of $\Phi(x)$. If $\hat{k}$ denotes the Fourier transform of $k$, then $\gamma_{\Phi}=\sigma^{2} \hat{k}$. In the usual setting, $k$ and $\hat{k}$ are related to each other by

$$
\hat{k}(\xi)=\frac{1}{2 \pi} \int_{\mathbb{R}^{2}} k(x) \mathrm{e}^{-i x \xi} \mathrm{d} x, \quad \xi \in \mathbb{R}^{2},
$$

and vice versa

$$
k(x)=\frac{1}{2 \pi} \int_{\mathbb{R}^{2}} \hat{k}(\xi) \mathrm{e}^{i x \xi} \mathrm{d} \xi, \quad x \in \mathbb{R}^{2} .
$$

For short, we use the symbols $\mathscr{F}$ and $\overline{\mathscr{F}}$ for the Fourier transform and its co-transform, which allows to rewrite $\hat{k}=\mathscr{F} k$ and $k=\mathscr{\mathscr { F }} \hat{k}$.
In isotropic case $\hat{k}$ depends only on the corresponding radial coordinate $\rho=\|\xi\|$, and $\hat{k}_{1}(\rho)=$ $\hat{k}(\xi)$ is the Fourier-Bessel transform of $k_{1}(r)$,

$$
\hat{k}_{1}(\rho)=\frac{1}{2 \pi} \int_{0}^{\infty} r k_{1}(r) \mathscr{J}_{0}(r \rho) \mathrm{d} r, \quad \rho \geq 0,
$$

where $\mathscr{J}_{0}$ is the Bessel function of the first kind of order 0. It is well known that the Fourier-Bessel transform of the exponential correlation function is

$$
\hat{k}_{1}(\rho)=\sqrt{\frac{2}{\pi}} \frac{\lambda}{\left(\lambda^{2}+\rho^{2}\right)^{3 / 2}}, \quad \rho \geq 0
$$

Graphs of this function are shown in Figs. 7 to 9 (red curves).

To be more flexible in modeling and characterizing paper formation, we introduce a generalized version of the exponential correlation function which depends on an additional positive parameter: the modified Bessel correlation function which is defined as

$$
k_{1}(r)=\frac{(\lambda r)^{v}}{2^{v-1} \Gamma(v)} \mathscr{K}_{v}(\lambda r), \quad r \geq 0,
$$

where $\Gamma$ denotes Euler's $\Gamma$-function and $\mathscr{K}_{v}$ is the modified Bessel function of second kind of order $v$. It is also here $k_{1}(r) \rightarrow 1$ as $r \downarrow 0$, and for $v=1 / 2$ the exponential correlation function is obtained. The spectral density of the modified Bessel correlation function is

$$
\hat{k}_{1}(\rho)=\frac{1}{2^{v-1} \Gamma(v)} \frac{\lambda^{2 v}}{\left(\lambda^{2}+\rho^{2}\right)^{v+1}}, \quad \rho \geq 0,
$$

(Yaglom, 1986, p. 368).

\section{A GEOMETRIC INTERPRETATION}

Bochner's theorem states that a spectral representation exists for every continuous, positive definite function, i.e., also for functions which are not necessarily covariance functions of GRFs. To give an example, we consider a macroscopically homogeneous and isotropic 2D Boolean model $\Xi$ with identically distributed and pairwise independent random segments (Stoyan et al., 1995). In fact, Boolean segment processes may serve as models for fiber systems of paper, where the fibers are 'scattered independently and uniformly' in the paper sheet. For example, Deng and Dodson (1994) and Provatas et al. (2000) used a Boolean segment processes for modeling fiber deposition. 


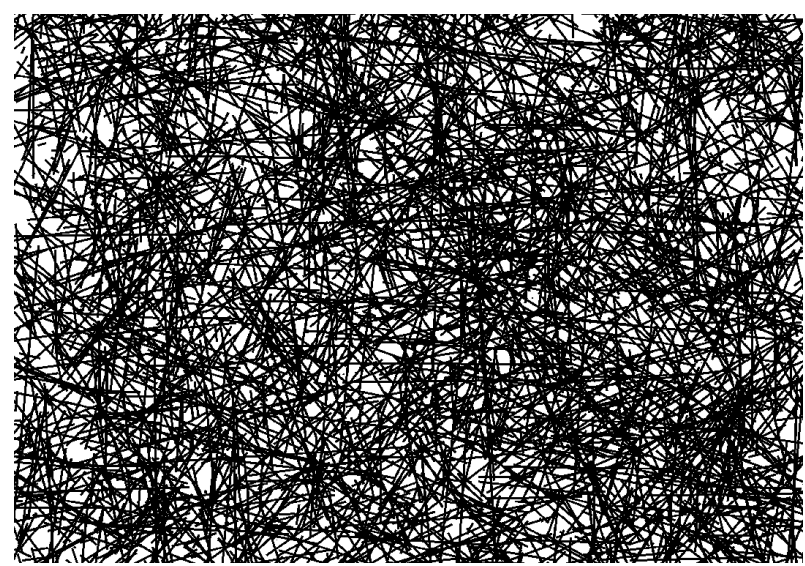

Fig. 4. A realization of an isotropic Boolean model with segments of uniformly distributed directions and exponentially distributed lengths, $1 / \alpha=2 \mathrm{~mm}, N_{A}=$ $10 \mathrm{~mm}^{-2}$; image size $20 \times 14.1 \mathrm{~mm}^{2}$.

In the following we assume that the length of the segments is exponentially distributed with the parameter $\alpha$, see Fig. 4 for a realization. Then the Boolean model $\Xi$ is uniquely characterized by the parameter $\alpha$ of the exponential distribution and the specific line length $L_{A}$, i.e., the mean of the total segment length per unit area. Notice that $1 / \alpha$ is the mean fiber length, and $N_{A}=\alpha L_{A}$ is the mean number of fibers per unit area. Then

$$
g(r)=1+\frac{\alpha}{N_{A} \pi r} \mathrm{e}^{-\alpha r}, \quad r>0,
$$

is the so-called pair correlation function of $\Xi$, defined as the density of the reduced second moment measure which is associated with random length measure $L$ of $\Xi$. An explanation and a general formula of the reduced second moment measure $K$ of Boolean segment processes is given in Stoyan et al. (1995), p. 186.

In order to obtain a GRF from the Boolean segment process, $\Xi$ is smoothed with a kernel function. Let $\kappa: \mathbb{R}^{2} \mapsto \mathbb{R}$ be a non-negative and bounded kernel function with $\int_{\mathbb{R}^{2}} \kappa(x) \mathrm{d} x=1$. By $\kappa^{*}(x)=\kappa(-x)$ we denote the reflection of $\kappa$, and $\kappa * f$ is the convolution of the a functions with the kernel $\kappa$. Furthermore, let $u: \mathbb{R} \mapsto \mathbb{R}^{2}$ be an arclength parametrization of a finite immersed curve $\varphi$ in $\mathbb{R}^{2}$, that is $\varphi=\{u(s): 0 \leq s \leq$ $\ell\}$, where $u$ is twice continuously differentiable and $\ell$ is the curve length. Similar to the convolution of functions we consider the convolution of a function with a measure (in our case the length measure associated with $\varphi$ ), where the convolution $\varphi * \kappa$ may be defined as

$$
(\varphi * \kappa)(x)=\int_{0}^{\ell} \kappa(x-u(s)) \mathrm{d} s, \quad x \in \mathbb{R}^{2},
$$

(Katznelson, 2004). Then $\Psi(x)=(\Xi * \kappa)(x)$ is a macroscopically homogeneous random field with $\mathbb{E} \Psi(x)=L_{A}$, but $\Psi(x)$ is of course not a GRF. (The random field $\Psi(x)$ forms a Poisson shot noise process with respect to a random response function, Matérn, 1986, p. 31.)

If we choose $\kappa$ such that it decreases sufficiently fast as $\|x\| \rightarrow \infty$, then from the central limit theorem (CLT) it follows that

$$
\Phi(x)=\lim _{N_{A} \rightarrow \infty} \frac{1}{N_{A}}\left(\Xi * \kappa-L_{A}\right)(x), \quad x \in \mathbb{R}^{2},
$$

forms a GRF with $\mu=0$ (Lane, 1984). The covariance function of $\Phi(x)$ is $\operatorname{cov}(x)=\alpha^{2}\left(\left(\kappa * \kappa^{*}\right) * h\right)(x)$, where $h(x)=g(\|x\|)-1$.

Let now $\left\{\kappa_{\varepsilon}\right\}_{\varepsilon>0}$ be a family of non-negative kernel functions of bounded support, $\kappa_{\varepsilon}(x)=0$ for $\|x\| \leq \frac{1}{\varepsilon}$. Then it follows that $\operatorname{cov}(x) \rightarrow \sigma^{2} h(x)$ as $\varepsilon \downarrow 0$ for all $x \in \mathbb{R}^{2}$.

In the line with the above, we are setting $h_{1}(r)=$ $g(r)-1$ and call $h_{1}$ the correlation function of the Boolean model $\Xi$. It holds that $h_{1}(r) \rightarrow \infty$ as $r \downarrow 0$, i.e., $h_{1}(r)$ is not a correlation function of a GRF. Nonetheless, the covariance measure corresponding to $h_{1}(r)$ is positive definite (Section 6.4 in Ohser and Schladitz, 2009) and, therefore, from Bochner's theorem it follows that there exists a Bartlett spectrum of $\Xi$. Moreover, the Bartlett spectrum has a density, i.e., the Bessel transform

$$
\hat{h}_{1}(\rho)=\frac{1}{\pi N_{A}} \frac{\alpha}{\sqrt{\alpha^{2}+\rho^{2}}}, \quad \rho \geq 0
$$

of $h_{1}$ exists, which is, up to a constant factor, the same as $\hat{k}_{1}$ given in Eq. 6 for $v=-\frac{1}{2}$. This is surprising, since the curve shape of $h_{1}$ basically differs from that of $k_{1}$ given in $\mathrm{Eg}$. 5 , where the parameter $\alpha$ plays the same role as the patameter $\lambda$ of the Bessel correlation function.

In other words, the GRF $\Phi(x)$ constructed by Eq. 7 inherits the second order properties of the Boolean model $\Xi$. This shows that there is a close relationship between 'independent and uniform scattering' of fibers in the plane (observable on a microscale) and paper formation (observable on a mesoscale), where the fiber mean length $1 / \alpha$ corresponds to the formation index. However, 'independent and uniform scattering' of fibers means that there is no tendency to form fiber clusters (flocks) induced e.g., by adhesion. Nevertheless, a significant formation is observable even if the fibers are 'independently and uniformly scattered', see Fig. 5 for an example. 


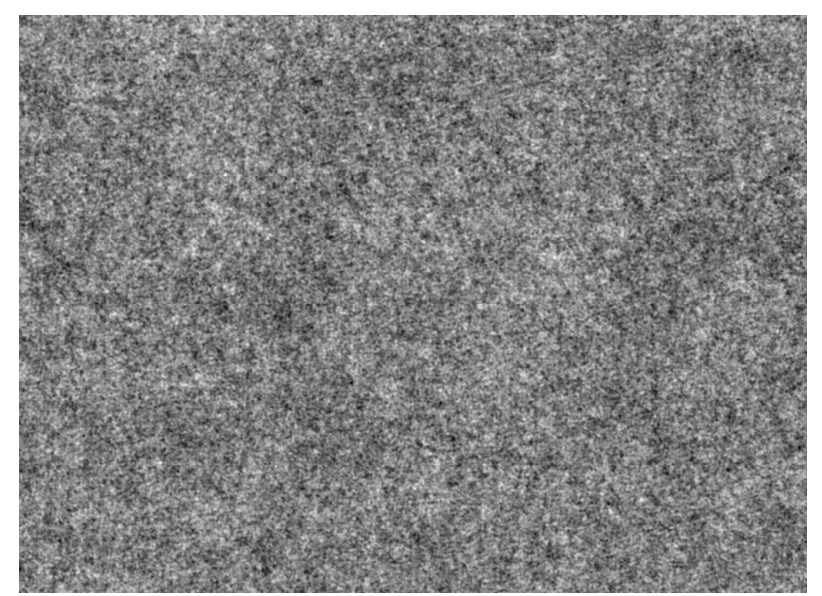

Fig. 5. A realization of a GRF based on an isotropic Boolean model with segments of exponentially distributed lengths, $1 / \alpha=2 \mathrm{~mm}$, where the smoothing kernel $\kappa$ is the probability density of the isotropic $2 D$ Gauss distribution with $\sigma=0.02 \mathrm{~mm}$. The width of the image is $102.4 \mathrm{~mm}$.

Finally we remark, that the geometric model for the fiber structure introduced above is neither the only possible nor the most simplest one. In particular, in Deng and Dodson (1994) one can find suggestions for more realistic distributions of the fiber length. However, the above model useful to derive a closed relationship between the fiber structure and the paper formation without any assumptions on flocculation.

\section{MONTE CARLO SIMULATION}

We follow the spectral approach developed by Shinozuka and Jan (1972) and others where realizations of a GRF are generated in the following two steps:

1. Let $u$ be a random number uniformly distributed on the interval $[0,1]$, and let $v$ be a random vector distributed with respect to the probability measure $\Gamma_{\Phi} / 2 \pi$ on $\mathbb{R}^{2}$. If $u$ and $v$ are independent, then

$$
\Psi_{x}=\sqrt{2} \cos (2 \pi u+v x), \quad x \in \mathbb{R}^{2},
$$

forms a macroscopically homogeneous and isotropic random field with mean $\mu=0$, variance $\sigma^{2}=1$ and correlation function $k$.

2. Let now $\Psi_{x}^{(1)}, \ldots, \Psi_{x}^{(m)}$ are mutually independent and identically distributed random fields with $\mu=$ $0, \sigma^{2}=1$ and $k$. Define

$$
\phi_{x}^{(m)}=\frac{1}{\sqrt{m}} \sum_{i=1}^{m} \Psi_{x}^{(i)}, \quad x \in \mathbb{R}^{2}
$$

Then the CLT it yields that

$$
\Phi(x)=\sigma \lim _{m \rightarrow \infty} \phi_{x}^{(m)}+\mu, \quad x \in \mathbb{R}^{2},
$$

is a macroscopically homogeneous and isotropic GRF with $\mu, \sigma^{2}$ and $k$.

Further details and an overview of alternative approaches are given in Lantuéjoul (2002).

But how large must $m$ be such that $\phi_{x}^{(m)}$ can be accepted as a realization of $\Phi(x)$ ? The usual way for a suitable choice of $m$ is based on the BerryEsseen inequality (Korolev and Shevtsova, 2010). For the realizations of the GRFs shown in Figs. 1 to 3 the number $m$ was empirically chosen as $m=4096$, which surely is large enough as $\hat{k}(\rho)$ vanishes rapidly at infinity, see also the remark in Lantuéjoul (2002), p. 192.

\section{ESTIMATION OF $\hat{k}$}

In this section we assume that the Bartlett spectrum $\Gamma_{\Phi}$ of the observed random field $\Phi(x)$ has a density. We start from an observation of the normalized random field $f(x)=(\Phi(x)-\mu) / \sigma$ having the expectation 0 and the variance 1 . In applications the field $f$ is observed through a compact window $W \subset \mathbb{R}^{2}$ with the indicator function $\mathbf{1}_{W}$ defined as $\mathbf{1}_{W}(x)=1$ if $x \in W$ and $\mathbf{1}_{W}(x)=0$ otherwise. This means that the masked function $f_{W}(x)=f(x) \cdot \mathbf{1}_{W}(x)$ is considered. One should keep in the mind that the image data can be seen as a realization of $f_{W}$, where $W$ is the (rectangular) image frame. Furthermore, we introduce a window function $c_{W}$ of $W$ defined as the autocorrelation function of the function $\mathbf{1}_{W}, c_{W}=\mathbf{1}_{W} * \mathbf{1}_{W}^{*}$, called the set covariance in Stoyan et al. (1995).

The function $c_{W}$ is bounded and of bounded support, and thus its Fourier transform $\hat{c}_{W}$ exists. From the Wiener-Khintchine theorem it follows that $\mathscr{F}\left(c_{W}\right.$. $k)=2 \pi \mathbb{E}\left|\hat{f}_{W}\right|^{2}$. The power spectrum $\mathbb{E}\left|\hat{f}_{W}\right|^{2}$ of $f_{W}$ is integrable, and hence the inverse Fourier transform $\overline{\mathscr{F}}$ can be applied, which yields

$$
c_{W} \cdot k=2 \pi \overline{\mathscr{F}}\left(\mathbb{E}\left|\hat{f}_{W}\right|^{2}\right) .
$$

Assume now that the origin belongs to $W$. Then $c_{W}$ is positive for all $x$ belonging to the interior of $W$, and it follows that

$$
\frac{2 \pi \overline{\mathscr{F}}\left(\left|f_{W}\right|^{2}\right)(x)}{c_{W}(x)}
$$

is an unbiased estimator of $k(x)$ for all $x$ in the interior of $W$.

In the isotropic case the rotation average of an estimation of $k$ can be performed (rotation around the origin), which gives an estimation of the radial 
function $k_{1}$. This leads to an estimation of the density $\hat{k}_{1}$ of the Bartlett-Spectrum using the 1-dimensional Fourier-Bessel transform as defined by Eq. 3 .

An overview of the whole estimation procedure is given in Fig. 6. Clearly, $k c_{W}$ can also be computed by auto-correlation (red marked path in Fig. 6).

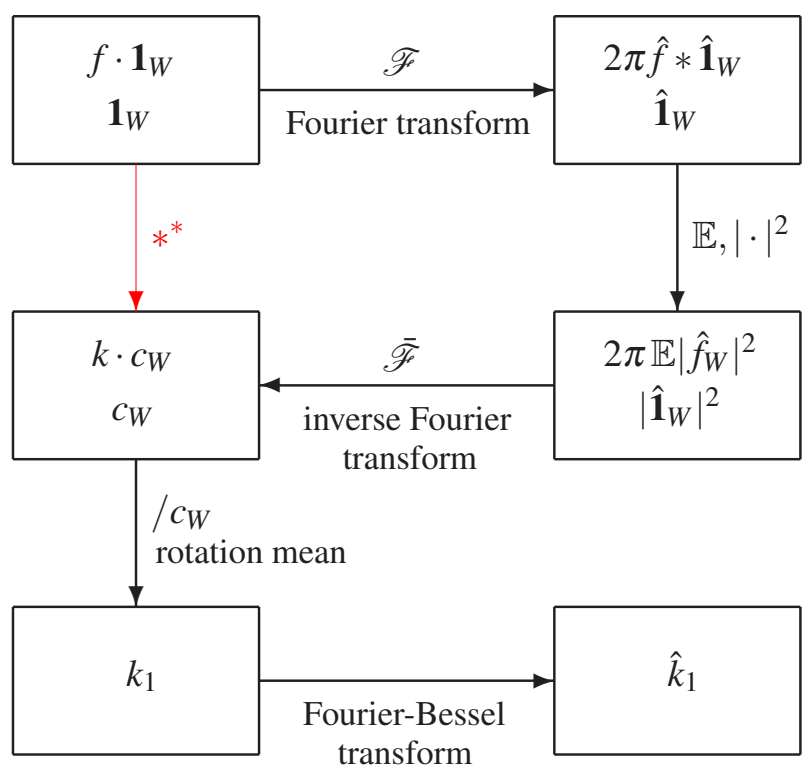

Fig. 6. Scheme of the computation of the density function $\hat{k}_{1}(\rho)$. The symbol $*^{*}$ stands for autocorrelation of functions (convolution with the reflected function).

It is well known that the correlation function $k$ can be computed using the Fast Fourier Transform (FFT) with a complexity in $\mathscr{O}(n \log n)$, where $n$ is the number of pixels of a image of $\Phi(x)$. Notice that also the window function can efficiently be computed via the inverse space using $c_{W}=\overline{\mathscr{F}}\left|\mathscr{F} \mathbf{1}_{W}\right|^{2}$. In the rectangular case, $c_{W}$ is explicitly known (Ohser and Mücklich, 2000 , p. 356). Furthermore, discrete version of the Bessel transform (necessary for computing $\hat{k}_{1}$ from $k_{1}$ ) can be based on numerical integration, e.g., by Romberg's rule.

Unfortunately, the assumption of periodicity in the discrete Fourier transform (dFT) causes an overlapping effect (edge effect). This effect can be eliminated by expanding the function $f_{W}$ to the window $2 W$, where $f_{W}$ is padded with zeros, that is $f_{2 W}(x)=f_{W}(x)$ if $x \in W$, and $f_{2 W}(x)=0$ if $x \in 2 W \backslash W$. This increases the pixel number to $4 n$, still the complexity of the FFT applied to $f_{2 W}$ belongs to $\mathscr{O}(n \log n)$, which is a considerable gain compared to the usual estimation of $k c_{W}$ based on auto-correlation, Fig. 6 (red path), which is of complexity $\mathscr{O}\left(n^{2}\right)$. Notice that data windowing using a $2 \mathrm{D}$ analogue of the Welch, Hann
(Hamming) or Bartlett window (Press et al., 2007) avoids any window expansion, but the unbiasedness of the estimator given by Eq. 8 gets lost.

The dFT (and its inverse) is usually based on a modified setting of the continuous Fourier transform. The main difference to be aware of, is that in Eqs. 1 and 2 the angular frequency $\omega=2 \pi \xi$ substitutes the frequency $\xi$. This has an impact on the scaling of the estimated spectral density.

Finally, we remark that sampling of $f$ on a homogeneous point lattice induces a sampling of $\hat{f}$ on the inverse lattice, where the relationship between the original lattice and its inverse is as follows: Let $U$ be a matrix of which the column vectors are forming a basis of the original lattice. Then the column vectors of the matrix $\left(U^{\prime}\right)^{-1}$ form a basis of the inverse lattice (Ohser and Schladitz, 2009, p. 66). In terms of a dFT applied to a 2D image with $n_{1} \cdot n_{2}$ pixels of size $a_{1} \cdot a_{2}$, the transformed image also consists of $n_{1} \cdot n_{2}$ pixels but of size $\hat{a}_{1} \cdot \hat{a}_{2}$, where $\hat{a}_{1}=1 /\left(n_{1} a_{1}\right)$ and $\hat{a}_{2}=1 /\left(n_{2} a_{2}\right)$.

\section{EXPERIMENTAL RESULTS}

The applicability of the method presented above is now demonstrated for three filter papers produced by wet laid cellulose fibers. The material No. 1 has a nominal grammage of $200 \mathrm{~g} / \mathrm{m}^{2}$ and a mean thickness of about $0.9 \mathrm{~mm}$, the material No. 2 is of $90 \mathrm{~g} / \mathrm{m}^{2}$ and about $0.25 \mathrm{~mm}$ thick, and the material No. 3 is of $170 \mathrm{~g} / \mathrm{m}^{2}$ and about $0.7 \mathrm{~mm}$ thick. The mean fiber length in these materials was much longer than $2 \mathrm{~mm}$.
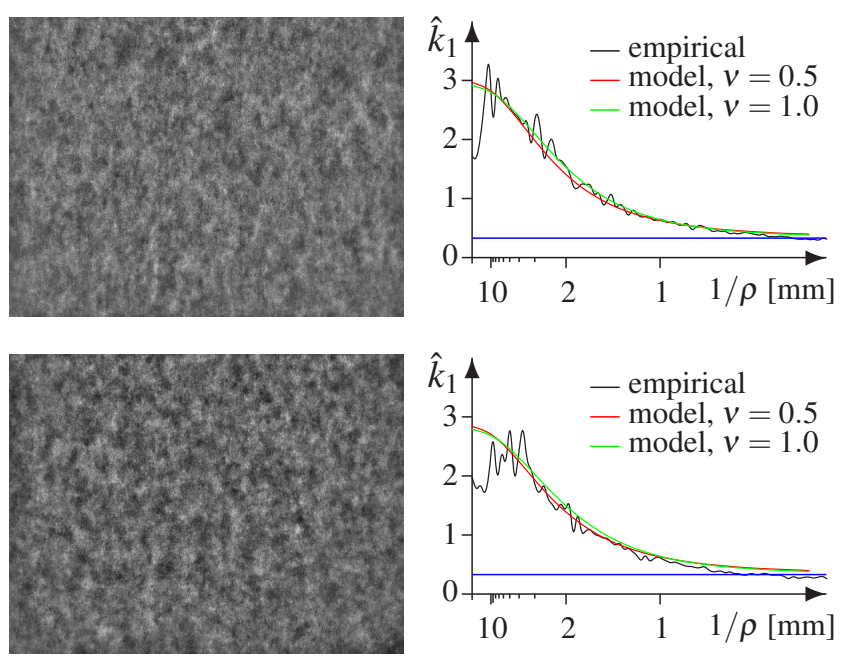

Fig. 7. Images showing the formations of the filter papers $\mathrm{Nr} \mathrm{la}$ (top) and $\mathrm{Nr} \mathrm{lb}$ (bottom), respectively, as well as the densities of their Bartlett spectra. 
In order to estimate the spectral density and to determine a formation index, various filter papers are scanned in the light transmission mode using a conventional CCD camera, see Figs. 7 to 9 (left) for examples. The 8-bit gray-tone images are of $1580 \times$ 1200 pixels with a lateral resolution of $0.177 \mathrm{~mm}$ per pixel and, thus, the effective image size amounts $279.66 \times 212.40 \mathrm{~mm}^{2}$. The wet laying process induces a slight sheet inhomogeneity appearing as a long wave shading in the corresponding images. This shading was corrected based on a reference image which was obtained by smoothing the image data using a large Gaussian filter with the parameter $\sigma=17.4 \mathrm{~mm}$, and where the reference image was subtracted from the original one.
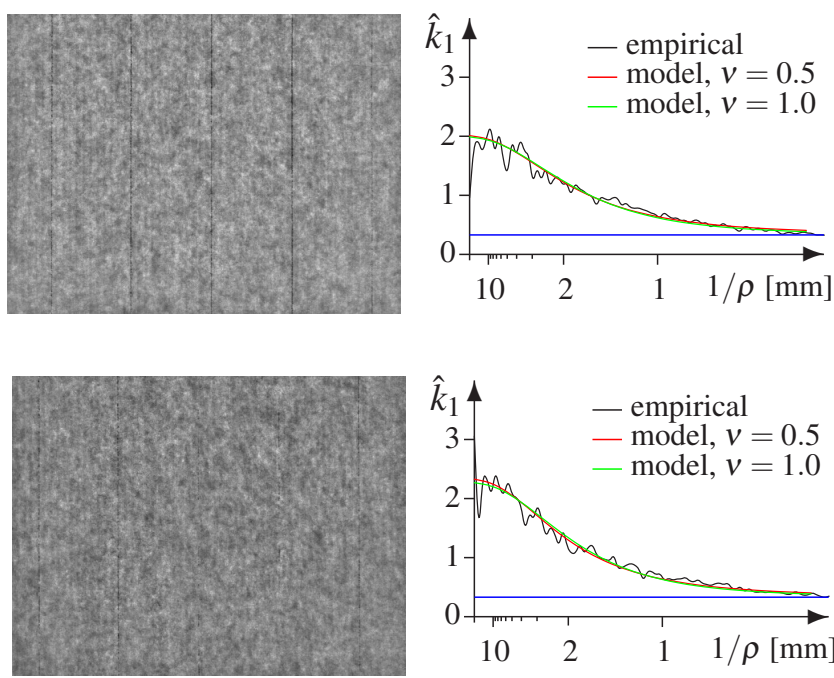

Fig. 8. The filter papers $\mathrm{Nr} 2 a$ (top) and $\mathrm{Nr} 2 b$ (bottom).

The function $\hat{k}_{1}$ is estimated from the image data using the method described in the previous section. The graphs of the estimates are shown in Figs. 7 to 9 (right), where $\hat{k}_{1}$ is given in $\mathrm{mm}^{2}$. Note that the relative small values of the empirical $\hat{k}_{1}$ for wave lengths $1 / \rho$ less than $10 \mathrm{~mm}$ might be a consequence of the applied shading correction. Generally, it is a hard problem to remove an unknown long wave shading under simultaneous keeping the spectrum of long waves in the real structure. Furthermore, because of data windowing, the fractions of long waves are estimated with a larger error than those of short waves. Nonetheless, analysis of realizations of GRFs with comparable spectral densities shows that for wave lengths less than $10 \mathrm{~mm}$ the function $\hat{k}_{1}$ is estimated from the image data with sufficiently small errors.
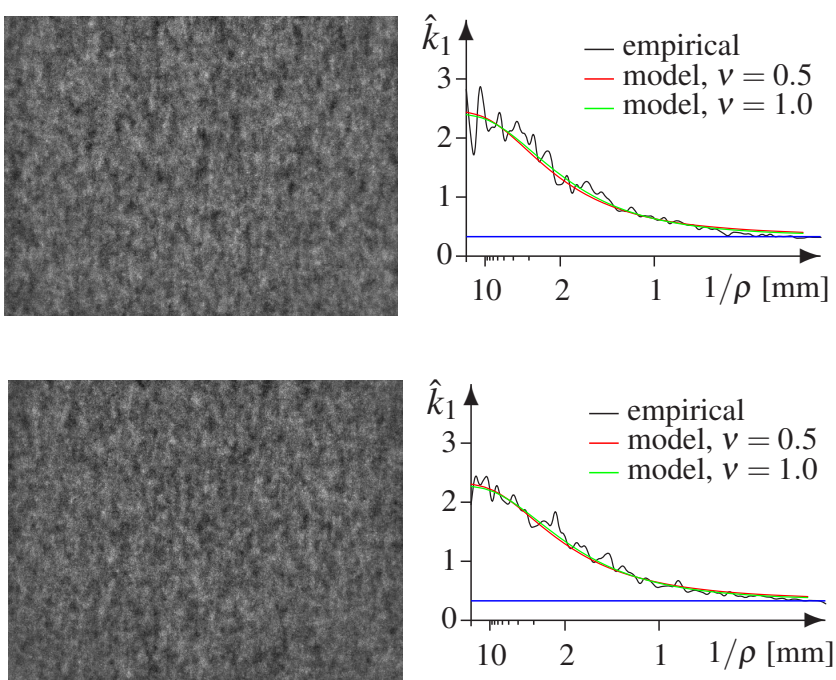

Fig. 9. The filter papers $\mathrm{Nr} 3 a$ (top) and $\mathrm{Nr} 3 b$ (bottom).

Obviously, there is a white noise observable as an additive constant $c$ of $\hat{k}_{1}$,

$$
c=\lim _{\rho \rightarrow \infty} \hat{k}(\rho) \approx 0.33 \mathrm{~mm}^{2}
$$

(the blue lines in Figs. 7 to 9, right). The reason for this is not clear. Probably, a considerable fraction of this noise comes from image acquisition.

Moreover, for fixed $v$ the theoretical function given by Eq. 6 was fitted to the experimental data for $\rho \leq$ $10 \mathrm{~mm}$, where the parameter $\lambda$ was estimated based on a least squares method (Figs. 7 to 9). The estimates of $\lambda$ are widely independent of $v$ (Tab. 1), and in the most cases the fit for $\mu=1$ is better than for $\mu=\frac{1}{2}$.

Table 1. The formation index $\beta$ and the numerical values $\lambda$ for the adapted function given by Eq. 6 .

\begin{tabular}{|c|c|c|c|c|}
\hline \multirow{2}{*}{$\begin{array}{c}\text { material } \\
\text { nr. }\end{array}$} & \multirow{2}{*}{$\begin{array}{c}\text { specimen } \\
\text { nr. }\end{array}$} & \multirow{2}{*}{$\begin{array}{c}\beta \\
{\left[\mathrm{mm}^{2}\right]}\end{array}$} & \multicolumn{2}{|c|}{$\lambda\left[\mathrm{mm}^{-1}\right]$} \\
\hline & & & $v=0.5$ & $v=1$ \\
\hline 1 & $1 \mathrm{a}$ & 2.6 & 0.550 & 0.557 \\
\hline 1 & $1 b$ & 2.3 & 0.564 & 0.568 \\
\hline 2 & $2 \mathrm{a}$ & 1.9 & 0.689 & 0.694 \\
\hline 2 & $2 b$ & 2.1 & 0.632 & 0.637 \\
\hline 3 & $3 a$ & 2.2 & 0.615 & 0.619 \\
\hline 3 & $3 b$ & 2.1 & 0.635 & 0.638 \\
\hline
\end{tabular}

Finally, a formation index $\beta$ is the determined as the mean of the density $\hat{k}_{1}$ for wave lengths between 2 and $5 \mathrm{~mm}$, which are relevant for industrial application. 


\section{DISCUSSION}

Throughout this article it is assumed that the paper structure is isotropic, but most papers produced on papermaking machines such as those based on the principles of the Fourdrinier Machine show a clear anisotropic formation, see also Schaffnit and Dodson (1993), Scharcanski and Dodson (1996, 2000) and Sampson (2009), where the anisotropy of formation is discussed in detail. Here we only remark that anisotropic paper formation corresponds to an anisotropic spectral density $\hat{k}(\xi)$, and from an estimate of $\hat{k}$ one can derive two quantities $\beta_{1}$ and $\beta_{2}$ describing the paper formation. Let $(\rho, \varphi)$ denote the polar coordinates of $\xi$ with $\rho \geq 0$ and $0 \leq \varphi<\pi$. Then the formation index $\beta_{1}$ can be computed from $\hat{k}\left(\rho, \varphi_{1}\right)$, where $\varphi_{1}$ is the processing direction of paper making, and $\beta_{2}$ is obtained from $\hat{k}\left(\rho, \varphi_{2}\right)$ for the direction $\varphi_{2}$ perpendicular to $\varphi_{1}$. Usually, $\beta_{1} \geq \beta_{2}$, and $\beta_{1}=\beta_{2}$ in the isotropic case.

For fixed $\varphi_{1}$ and $\varphi_{2}$, the functions $\hat{k}_{1}^{\perp}(\rho)=$ $\hat{k}\left(\rho, \varphi_{1}\right)$ and $\hat{k}_{2}^{\perp}(\rho)=\hat{k}\left(\rho, \varphi_{2}\right)$ can be seen as planar sections profiles of the spectral density $\hat{k}(x)$. From the projection slice theorem (Kuba and Hermann, 2008) it immediately follows that $\hat{k}_{1}^{\perp}(\rho)$ and $\hat{k}_{2}^{\perp}(\rho)$ can be obtained as a cosine transform of the orthogonal projections $k_{1}^{\perp}(r)$ resp. $k_{2}^{\perp}(r)$ of the estimated correlation function $k(x)$ onto the corresponding section planes, i.e., the rotation mean in the scheme of Fig. 6 is replaced with the orthogonal projections onto the two section planes, and the Fourier-Bessel transform is now a simple cosine transform.

As pointed out in this article, there is a 'basic formation' related to an 'independent and uniform scattering' of the paper fibers, and even this 'basic formation' can probably not be effected by technological measures. This means that the possibility to reduce paper formation by an improved paper making technology is limited. For a paper with a given formation, the question is as follows: What is the difference between the given and the 'basic' formation? Unfortunately, the 'basic formation' can be estimated only roughly from the distribution of the fiber lengths and until now there is no safe method to find out whether the formation of a paper can be reduced or not. Notice that just Deng and Dodson stated in their monograph "that the power spectrum can give information on the flock sizes, by isolating the variance components between two scales for inspection zones" (Deng and Dodson, 1994, p. 107).

The computation of the formation index from images of transmitted light via frequency space is very efficient. However, the results from different laboratories are comparable only under the condition that the spectral density of the intensity of the transmitted light is (nearly) the same as the spectral density of the local paper grammage. Thus, when implementing a laboratory system for industrial quality control one should take care of the wavelength of the applied light, the homogeneity of illumination, the image acquisition, a possible inhomogeneity of the paper, and the edge effects involved in the computation of the spectral density. It is very helpful to make tests as the following one: the increase of the paper weight should not influence the formation and, therefore, the paper formation of a single paper sheet must be the same as that of a double sheet (both sheets of the same formation and one sheet on top of the other). Furthermore, the estimation of the spectral density should be robust with respect to variations of the lateral resolution, i.e., varying the pixel size (in the range from 0.05 to $0.2 \mathrm{~mm}$ ) should lead to only small changes in the estimated spectral density. Finally, the size of the paper sheet (i.e., the size of the window $W$ ) should be large enough such that the statistical errors of the estimates are limited. From our experience we can say that an A4-sheet is sufficient. More precisely, let $\Phi(x)$ be a Gaussian random field with an exponential correlation function, $\lambda \gtrsim 0.5 \mathrm{~mm}^{-1}$, observed through a rectangular window $W$ of the size $210 \times 297 \mathrm{~mm}^{2}$, then simulation studies show that the relative statistical error of estimation of $\hat{k}_{1}(\rho)$ is less than $5 \%$ for all wave lengths $1 / \rho \leq 5 \mathrm{~mm}$.

\section{ACKNOWLEDGEMENT}

This work was supported by the German Federal Ministry of Education and Research (BMBF) under grant MNT/03MS603C/AMiNa.

\section{REFERENCES}

Abrahamsen P (1985). A review of random fields an correlation functions, 2nd ed. Tech. Rep. 314, Blindern, Oslo.

Adler RJ (1981). The geometry of random fields. New York: John Wiley \& Sons.

Adler RJ, Taylor J (2007). Random fields and geometry. New York: Springer.

Alava M, Niskanen K (2006). The physics of paper. Rep Prog Phys 69:669-723.

Antoine C (2000). GRACE, a new tool to simulate paper optical properties. In: van Lieshout $M$, ed., New characterisation models for fibres, pulp and paper. Joint publication of the COST action E11 working group paper. 
Bergeron M, Bouley R, Drouin B, Gagnon R (1988). Simultaneous moisture and basis weight measurement. Pulp and Paper Magazine of Canada 89:173-6.

Boeckerman PA (1992). Meeting the special requirements for on-line basis weight measurements of lightweight nonwoven fabrics. Tappi J 75:166-72.

Burt BJ, Adelson EH (1983). The Laplacian pyramid as a compact image code. IEEE Trans Comm 31:532-40.

Cherkassky A (1998). Analysis and simulation of nonwoven irregularity and nonhomogeneity. Textile Res J 68:242-53.

Cherkassky A (1999). Evaluating nonwoven fabric irregularity on the basis of Linnik functionals. Textile Res J 69:701-8.

Chinga-Carrasco G (2009). Exploring the multi-scale structure of printing paper - a review of modern technology. J Microsc 234:211-42.

Cresson TM (1988). The sensing, analysis and simulation of paper formation. Ph.D. thesis, State University, New York.

Cresson TM, Luner P (1990a). The characterization of paper formation, Part 2: The texture analysis of paper formation. Tappi J 12:175-84.

Cresson TM, Luner P (1990b). Description of the spatial gray level dependence method algorithm. Tappi J $12: 220-2$.

Deng M, Dodson C (1994). Paper: an engineered stochastic structure. Tappi Press.

Drouin B, Gagnon R, Cheam C, Silvy J (2001). A new way for testing paper sheet formation. Compos Sci Techn 61:389-91.

Durst M, Klein GM, Moser N, Trautmann P (2007). Filtration und Separation in der Automobiltechnik. Chem Ing Techn 79:1845-60.

Farnood RR, Dodson CTJ, Loewen SR (1995). Modeling flocculation. Part I: Random disc model. J Pulp and Paper Sci 21:J348-56.

Farrington TE (1988). Soft X-ray imaging can be used to asses sheet formation and quality. Tappi J 71:140-4.

Gregersen ØW, Niskanen K (2000). Measurement and simulation of paper 3D-structure. In: van Lieshout M, ed., New characterisation models for fibres, pulp and paper. Joint publication of the COST action E11 working group paper.

I'Anson S (1995). Identification of periodic marks in paper and board my image analysis using two-dimensional fast Fourier transforms. Tappi J 78:97-106.

I'Anson S, Sampson WW (2003). Determination of spatial domain formation statistics using fast Fourier transform. Paperija Puu 85:403-8.

ISO 4046(E/F) (2012). International standard on paper, board, pulps and related terms - Vocabularity, Part 15. ISO Copyright Office, Geneva.

Kallmes OJ (1984). The measurement of formation. Tappi J 67:117-26.

Katznelson Y (2004). An introduction to harmonic analysis. Cambridge Mathematical Library. Cambridge: Cambridge University Press, 3rd ed.

Koch K, Ohser J, Schladitz K (2003). Spectral theory for random closed sets and estimating the covariance via frequency space. Adv Appl Prob 35:603-13.

Korolev VY, Shevtsova IG (2010). On the upper bound for the absolute constant in the Berry-Esseen inequality. Th Prob Appl 54:638-58.

Kuba A, Hermann G (2008). Some mathematical concepts for tomographic reconstruction. In: Banhart J, ed., Advanced tomographic methods in materials science and engineering. Oxford: Oxford University Press, 1936.

Lane JA (1984). The central limit theorem for the Poisson shot-noise process. J Appl Prob 21:287-301.

Lantuéjoul C (2002). Geostatistical simulation - models and algorithms. Berlin: Springer.

Lien HC, Liu CH (2006). A method of inspecting non-woven basis weight using the exponential law of absorption and image processing. Textile Res J 76:547-58.

Matérn B (1986). Spatial variation. Berlin: Lecture notes in statistics 36, Springer-Verlag.

McDonald JD, Rarrell WR, Stevens RK, Hussain SM, Roche AA (1986). Using an on-line light transmission gauge to identify the source of grammage variations. J Pulp and Paper Sci 12:J1-9.

Norman B (1986). The formation of paper sheets, Chapter 6. In: Bristow AJ, Kolseth P, eds, Paper, structure and properties, vol. 8 of Int Fiber Sci and Techn Series. Marcel Dekker.

Norman B, Wahren D (1974). The measurement of mass distribution in paper sheets using a beta radiograph method. Svensk Papperstilding 11:397-406.

Norman B, Wahren D (1976). Mass distribution and sheet properties of paper. In: Fundamental properties of paper to its end uses, Trans. Symp. London: B.P.B.I. F.

Ohser J, Mücklich F (2000). Statistical analysis of microstructures in materials science. Chichester, New York: J Wiley \& Sons.

Ohser J, Schladitz K (2009). 3D images of materials structures - processing and analysis. Weinheim, Berlin: Wiley VCH.

Pourdeyhimi B, Kohel L (2002). Area-based strategy for determining web uniformity. Textile Res J 72:1065-72.

Praast H, Göttsching L (1991). Formation graphischer 
Papiere. Das Papier 45:333-47.

Press WH, Teukolsky SA, Vetterling WT, Flannery BP (2007). Numerical recipes - The art of scientific computating, 3rd Ed. Cambridge University Press.

Provatas N, Alava MJ, Ala-Nissila T (1996). Density correlations in paper. Phys Rev E 54:R36-8.

Provatas N, Haataja M, Asikainen J, Majaniemi S, Alava M, Ala-Nissila T (2000). Fiber deposition models in two and three dimensions. Colloids Surfaces A 165:209-29.

Robertson AA (1956). The measurement of paper formation. Pulp and Paper Magazine of Canada 55:119-27.

Sampson WW (2009). Modelling stochastic fibrous materials with mathematica. Engineering materials and processes. London: Springer.

Sara H (1978). The characterization and measurement of paper formation with standard deviation and power spectrum. Ph.D. thesis, Helsinki University, Helsinki.

Schaffnit C, Dodson CTJ (1993). A new analysis of fiber orientation effects on paper formation. Paperija Puu 75:68-75.

Scharcanski J (2006). Stochastic texture analysis for measuring sheet formation variability in the industry. IEEE Trans Instrum Meas 55:1778-85.

Scharcanski J, Dodson CTJ (1996). Texture analysis for estimating spatial variability and anisotropy in planar structures. Opt Eng J 35:2302-8.

Scharcanski J, Dodson CTJ (2000). Stochastic texture image estimators for local spatial anisitropy and its variability. IEEE Trans Instrum Meas 49:971-9.

Scholz M, Claus B (1999). Analysis and simulation of nonwoven textures. Z Angew Math Mech 79:237-40.

Shinozuka M, Jan CM (1972). Digital simulation of random processes and its applications. J Sound Vibration 25:111-28.

Stoyan D, Kendall W, Mecke J (1995). Stochastic geometry and its applications. Chichester: J. Wiley \& Sons, 2nd ed.

Van den Akker JA (1949). Scattering and absorption of light in paper and other diffusing media. Tappi J 32:498-501.

Wang X, Georganas ND, Petriu EM (2011). Fabric texture analysis using computer vision techniques. IEEE Trans Instrum Meas 60:44-56.

Waterhouse JF, Hall MS, Ellis RL (1991). Strength improvement and failure mechanisms, 2. Formation. Tech Rep Project 3469, Report II, Institute of Paper Science and Technology (API), Atlanta, Gorgia.

Xu B (1996). Identifying fabric structures with fast Fourier transform techniques. Textile Res J 66:496-506.

Yaglom AM (1986). Correlation theory of stationary and related random functions I: Basic results. Springer Series in Statistics. New York: Springer.

Yuhara T, Hasuike M, Murakami K (1986). Application of image processing technique for analysis on sheet structure of paper, Part 1: quantitative evaluation of 2dimensional mass distribution. Japan Tappi 40:85-91. 